\title{
Declarative Assembly of Web Applications from Predefined Concepts
}

\author{
Santiago Perez De Rosso \\ MIT CSAIL \\ Cambridge, MA, USA \\ sperezde@csail.mit.edu
}

\author{
Daniel Jackson \\ MIT CSAIL \\ Cambridge, MA, USA \\ dnj@mit.edu
}

\author{
Maryam Archie \\ MIT CSAIL \\ Cambridge, MA, USA \\ marchie@mit.edu
}

\author{
Czarina Lao \\ MIT CSAIL \\ Cambridge, MA, USA \\ mcslao@mit.edu
}

\author{
Barry A. McNamara III \\ MIT CSAIL \\ Cambridge, MA, USA \\ barryam3@mit.edu
}

\begin{abstract}
A new approach to web application development is presented, in which an application is constructed by configuring and composing concepts drawn from a catalog developed by experts.

A concept is a self-contained, reusable increment of functionality. Each concept includes both front-end and back-end functionality, and exports a collection of components-fullstack GUI elements, backed by application logic and database storage.

To build an app, the developer imports concepts from the catalog, tunes them to fit the application's particular needs via configuration variables, and links concept components together to create pages. Components of different concepts may be executed independently, or bound together declaratively with dataflows and synchronization. The instantiation, configuration, linking and binding of components is all expressed in a simple template language that extends HTML.

The approach has been implemented in a platform called Déjà $\mathrm{Vu}$, which we outline and compare to conventional web application architectures. We describe a case study in which a collection of applications previously built as team projects for a web programming course were replicated in Déjà Vu. Preliminary results validate our hypothesis, suggesting that a variety of non-trivial applications can be built from a repository of generic concepts.
\end{abstract}

Permission to make digital or hard copies of all or part of this work for personal or classroom use is granted without fee provided that copies are not made or distributed for profit or commercial advantage and that copies bear this notice and the full citation on the first page. Copyrights for components of this work owned by others than the author(s) must be honored. Abstracting with credit is permitted. To copy otherwise, or republish, to post on servers or to redistribute to lists, requires prior specific permission and/or a fee. Request permissions from permissions@acm.org. Onward! '19, October 23-24, 2019, Athens, Greece

(C) 2019 Copyright held by the owner/author(s). Publication rights licensed to ACM.

ACM ISBN 978-1-4503-6995-4/19/10 . \$15.00

https://doi.org/10.1145/3359591.3359728
CCS Concepts - Software and its engineering $\rightarrow \mathbf{A b}$ straction, modeling and modularity; Organizing principles for web applications; Very high level languages; Modules / packages; Designing software.

Keywords application development, web applications, concepts, design, software design, modularity

\section{ACM Reference Format:}

Santiago Perez De Rosso, Daniel Jackson, Maryam Archie, Czarina Lao, and Barry A. McNamara III. 2019. Declarative Assembly of Web Applications from Predefined Concepts. In Proceedings of the 2019 ACM SIGPLAN International Symposium on New Ideas, New Paradigms, and Reflections on Programming and Software (Onward! '19), October 23-24, 2019, Athens, Greece. ACM, New York, NY, USA, 15 pages. https://doi.org/10.1145/3359591.3359728

\section{Introduction}

\subsection{Motivation}

As a user, you might have noticed the fundamental similarities between the many applications you use on a day-to-day basis. Maybe it was the day you were scrolling through your Facebook news feed and then through your Twitter feed; or giving a 5-star review to a restaurant on Yelp, and then to a book on Amazon. And just as you, as a user, experience the same rating concept in different variants in multiple applications, so the developers of those applications are, for the most part, implementing that concept afresh as if it had never been implemented before.

In each of these cases, the developer may be implementing something slightly different: a rating of a post in one case, and of a user in another. The premise of this paper, however, is that these are merely instantiations of the same generic concept, and that if this genericity could be captured, application development could be recast as a combination of pre-existing concepts in novel ways. This might then allow applications to be assembled with much less effort, since the core functionality of the individual concepts would not need to be repeatedly rebuilt. 
Our paper shows how this can be done in the context of a new platform called Déjà Vu. An application is constructed by assembling concepts that are implemented as reusable, full-stack modules. The assembly requires no procedural code: concept components are glued together by declarative bindings that ensure appropriate synchronization and dataflow. These bindings are expressed in a simple HTML-like template language, augmenting conventional layout declarations that determine which user interface widgets from which concepts are used, and how they are placed on the page. The net result, as we demonstrate through a case study, is that fairly complex applications can be built with little effort.

\subsection{Building Web Apps: The Status Quo}

Suppose you wanted a simple clone of Hacker News ${ }^{1}$, called Slacker News $(S N)$, in which registered users can post links, comment on posts, and upvote posts or comments. How would you build such an app?

General-Purpose Tools. You might use general-purpose programming languages, coupled with a web framework, some web libraries and a database. Even if you are an advanced web developer, it would take days to build such an app. You could perhaps save time by using full-stack components provided by a web API service. For example, you could use Disqus ${ }^{2}$ to implement comments. If the commenting functionality were isolated from the rest of the app's functionality, this could be done with very little effort (usually by just including some JavaScript and writing a little HTML). But $S N$ intertwines commenting with upvoting and posting: you want Disqus's users to be the same users that can post links, and you want users to be able to upvote comments as well as posts. Even if the API were flexible enough for you to be able to pull off such an integration, it would likely require you to open up the full-stack black box, and write server-side code. For example, you might need to create a notification hook so that your app is notified when a comment is created, so that it can be given an initial score.

Content Management Systems. Another option would be to use a content management system (CMS) such as Drupal ${ }^{3}$ or WordPress ${ }^{4}$. A CMS supports the creation of websites whose functionality primarily involves reading and writing content. Many CMSs provide a large suite of plug-ins that allow a website to be extended with new features. Like our concepts, these plug-ins are full-stack, and importing and configuring them is usually straightforward. But because plug-ins lack a generic composition mechanism, they can usually only be combined in certain predefined ways. More

\footnotetext{
${ }^{1}$ https://news.ycombinator.com/

${ }^{2}$ https://disqus.com/

${ }^{3}$ https://www.drupal.org

${ }^{4}$ https://wordpress.com/
}

application-specific combinations typically require modifying server-side CMS code. For example, you might be able to include a "comment" plug-in in your app, but if it's not designed to work with the "upvote" plug-in, you'd have to write complex glue code.

Low-Code Tools. A third option is to use a low-code development platform (e.g., Microsoft Power Apps ${ }^{5}$ ), or one of the many tools and languages for end-user programming of datacentric apps (e.g., [19, 30]). These tools typically offer a visual interface and domain-specific languages to specify a schema, queries, updates, and views. For standard CRUD (createread-update-delete) functionality, these platforms can work well, and they allow arbitrary customization of queries and updates (which our Déjà Vu platform does not). Code for handling the mechanics of GUI elements, data storage, server requests, etc., is provided and hidden, and this is a great help. But unlike Déjà $\mathrm{Vu}$, which provides implementations of rich behavior, a low-code approach will still require each new concept to be implemented anew. Moreover, functionality that is not expressible in the language-because it involves some algorithmic complexity, or a more complex user interface widget-must be provided by pre-built plug-ins. For example, $S N$ might require posts to be recommended to users based on the preferences they expressed in their upvoting of other posts; this might be provided as a machine learning plug-in. As with CMSs, such plug-ins can only be composed in ad hoc ways, and may require complex glue code.

\subsection{Déjà Vu's Approach}

Concept as Modules. In our approach, concepts are the building blocks of applications. A concept is a self-contained, reusable increment of functionality that is motivated by a purpose defined in terms of the needs of an end user [17]. For example, a comment concept manages the creation and display of comments, and a scoring concept lets users assign scores to items. Concepts are more fine-grained than traditional plug-ins or microservices, which would likely, for example, treat all the concepts associated with user reviewing (such as scoring, comments and recommendations) as part of a single plug-in or service.

Full-Stack Modules. A Déjà Vu application is a set of concept instances that run in parallel. Each instance is a fullstack service in its own right, with front-end GUI components, a back-end server and data storage, and all the code necessary to coordinate them. ${ }^{6}$ By default, these services run entirely independently of one another, in complete isolation. ${ }^{7}$ They share no data and do not exchange messages

\footnotetext{
$\overline{5}$ https://powerapps.microsoft.com

${ }^{6}$ The back-end server and data storage might be replicated for scalability.

${ }^{7}$ The services can run on separate machines or be co-located with our platform's runtime system for performance.
} 
with one other. Abstractly, a concept instance is a state machine that changes state only in response to actions issued by the user through the front-end.

Data Integration Through Shared IDs. Often, concept instances hold distinct views of objects that can be thought of as shared. For example, when a scoring concept is combined with a commenting concept, one can imagine a single object having both a scoring view (containing its scoring value) and a commenting view (containing the textual comment). But, as explained above, there is no explicit sharing of data. Instead, common identifiers are used to implicitly bind the distinct views. This is achieved using a special platform function that generates an identifier, which is then passed to the two concept instances, ensuring that the objects they hold will subsequently be associated.

The binding of identifiers between concepts is the source of polymorphism in Déjà Vu. A more conventional scheme, in which concepts had type parameters that had to be instantiated, would be less flexible and would impose a greater burden on the programmer.

Declarative Client-Side Synchronization. Each GUI component of a concept contains a front-end widget and a set of corresponding server-side actions. To connect the different concepts-making their actions occur together, and passing data from one to another-the programmer references the GUI components of multiple concepts in a client-side file, along with declarative bindings that indicate which actions of the components are synchronized, and how their inputs and outputs are related.

Action synchronization is transactional, ensuring that all or none of the concept server-side actions occur. Of course, not all components need to be synchronized. Components can also be placed on a web page so that they run independently of one another.

The same transaction mechanism can be used for security. Concepts are provided for user authentication and access control whose actions are designed to fail when access is denied. By synchronizing their actions with the actions of other concept instances, you can ensure that certain actions only occur when they should be permitted.

\subsection{Contributions}

The idea that software should be built from prefabricated parts dates back at least to 1968 [20]. Since then, many mechanisms to support large-scale software reuse have been developed. What's new in this work is what the parts arefull-stack implementations of concepts-and how they are put together-by declarative synchronization.

This paper makes the following contributions:

- A new approach to application development, showing how functionality can be understood as a composition of concepts drawn from a catalog.

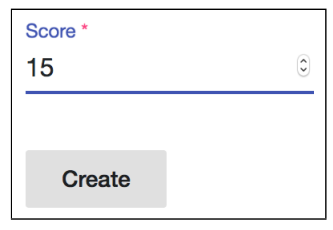

(a) create-score with targetId input "ben" and sourceId "alyssa"

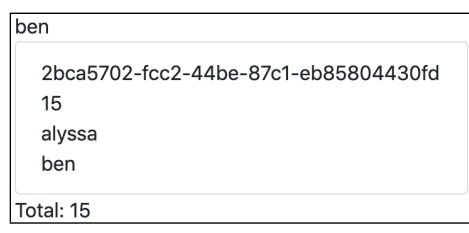

(b) show-target with id input "ben" showing the score created in Fig. 1a and ben's total score
Figure 1. Screenshots of two components of Scoring

- A new platform that supports independent development of concepts, a simple template language for instantiating and composing them, and an infrastructure for ensuring transactional semantics.

- A case study in which we used the platform to build a suite of non-trivial sample applications.

\section{Building Apps with Déjà Vu}

To see what using Déjà $\mathrm{Vu}$ is like, let's consider building Slacker News $(S N)$ (see §1.2). This section is written from the perspective of the Déjà Vu user; we talk about how to author concepts later in §3.4. To build apps with Déjà Vu, users include and configure concepts (\$2.1) and create app components by linking components (\$2.2). Users can also provide CSS to customize the appearance of the application.

\subsection{Including and Configuring Concepts}

Choosing Concepts. The process of building a Déjà Vu app begins by navigating the catalog of concepts to find the concepts that provide the functionality you need for your app. The documentation accompanying a concept includes information about the configuration options and the exported components. Fig. 1 shows some components of Scoring. Concept components control a patch of the screen, are interactive, and can read and write back-end data. They also have input and output properties.

SNuses Authentication to handle user authentication, Comment to comment on posts and reply to comments, and Scoring twice: for keeping track of upvotes on both posts and on comments separately. It also uses Property-which provides a data-model-defining facility for simple CRUD behavior-to save a post's author, title, and url.

Including Concepts. The concepts used by the app are specified in the app's JSON config file (Fig. 2, lines 3-22). The usedConcepts object has one key-value pair per concept instance. The key (e.g., "post" on line 6) determines the name that is going to be used in the HTML to refer to that instance. The value is another object with two optional key-value pairs: name for providing the name of the concept to be instantiated (e.g., "Property" on line 7), and config for specifying the configuring options for the concept instance (e.g., the 


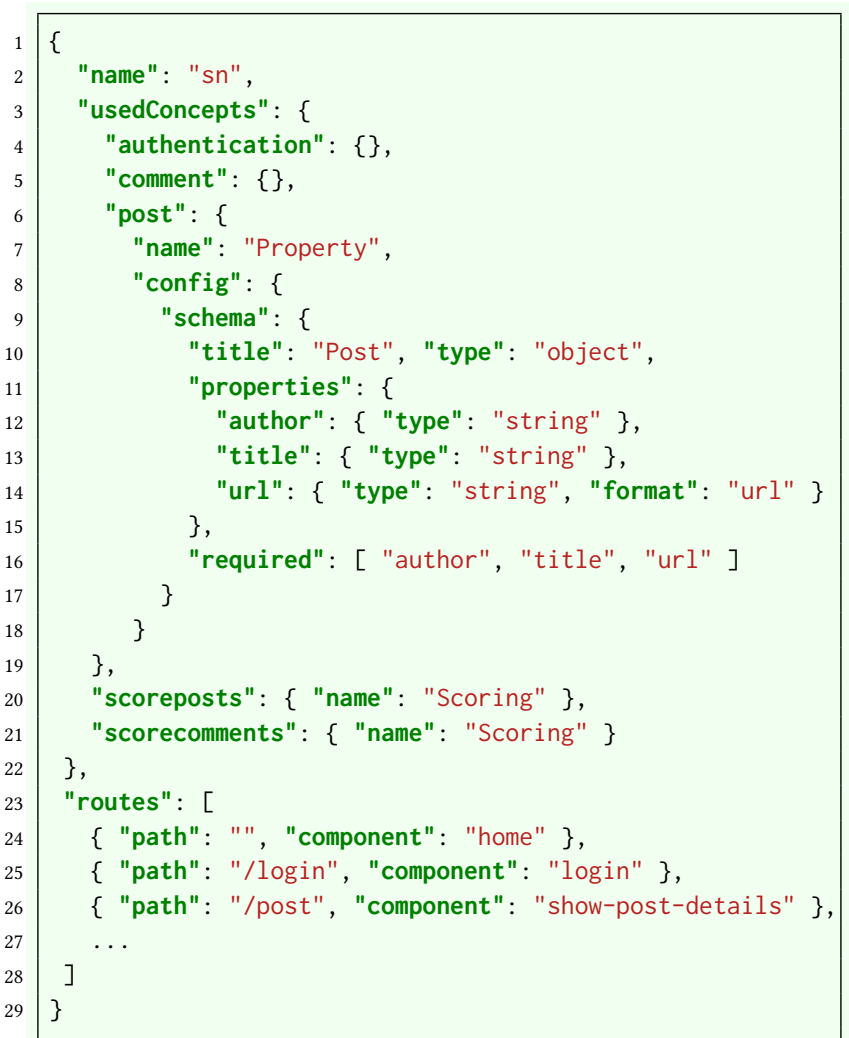

Figure 2. Excerpt of $S N$ s configuration file

object in lines 8-18). If no concept name is provided, the concept instantiated is the one with name equal to the instance name. Thus, for example, the concept to be instantiated for "authentication" is Authentication (line 4). If no configuration object is given, the default configuration for that concept is used. The format of the values of configuration options is also JSON.

Configuring Concepts. In $S N$, we only have to configure Property. Property accepts a configuration variable (schema) that expects a JSON Schema ${ }^{8}$ to describe the objects it will be saving. We use schema to specify the type of properties we expect our objects to have. In $S N$, our objects are "posts" (line 10), and we expect them to have an author, title, and a url (lines 11-15). The effect of this is that when we include a component from Property, such as create-object, the component will allow the user to input only those fieldsauthor, title, and url. Moreover, since we specified that the format of the url field is url (line 14) and that the fields author, title, and url are required (line 16), create-object will expect the user to provide a value for each one and check that the value given for the url field is a valid URL. If the

\footnotetext{
${ }^{8}$ https://json-schema.org/
}

user doesn't provide a value for each field, or if the value for url is invalid, create-object will show an error message.

Other App Configuration. In the app's config file, we also define the name (Fig. 2, line 2) and routes (lines 23-28) of our app. Each route maps a URL path to a component. A component that is accessible via URL is a page. $S N$ 's homepage is the component home (line 24) because path is empty. If the user navigates to /login, the login component will be shown (line 25) and if they navigate to /post, the show-post-details component will be shown (line 26).

\subsection{Linking Components}

Each app component is written in a separate HTML file. Fig. 3 shows excerpts of the code for $S N$ 's submit-post and show-post components, together with a screenshot of how they appear to users. Our template language looks, by design, similar to other template languages. To create an app component, users include components (\$2.2.1) and synchronize them to implement the desired functionality (§2.2.2).

\subsubsection{Including Components}

Including Components. An app component can contain other components, which can be concept components or app components. A concept component is a component that is defined and exported by a concept. An app component, on the other hand, is defined by the app developer and it is part of the app being developed. Components are included as if they were HTML elements, with the tag given by the concept instance or app name, followed by the component name. Thus, submit-post (Fig. 3a) includes one app component, navbar (line 2); two concept components, create-object of the "post" instance of Property (lines 5-8), and create-score of the "scoreposts" instance of Scoring (lines 9-11); and one built-in component, dv.gen-id (line 4), which generates a unique ID.

I/O Binding. Inputs to a component are bound with the syntax property=expr. Template expressions can include literal values, app component inputs, outputs from included components, and standard operators. Components can be fired repeatedly, and the output properties hold the values from the last execution. This is how a selector widget such as a dropdown would typically be connected to another component: the dropdown sets an output property every time it is activated containing the choice the user made, which is then bound to the input property of components that use that choice.

Some input properties are for customizing appearance and have no impact on the behavior of the component. For example, as a result of setting buttonLabel to "Submit" on line 7, Fig. 3a, create-object's button will carry the label "Submit" instead of the default button label "Create Post". The hidden property of show-object (Fig. 3b, line 2) indicates that the component should be activated but not visible. Thus 

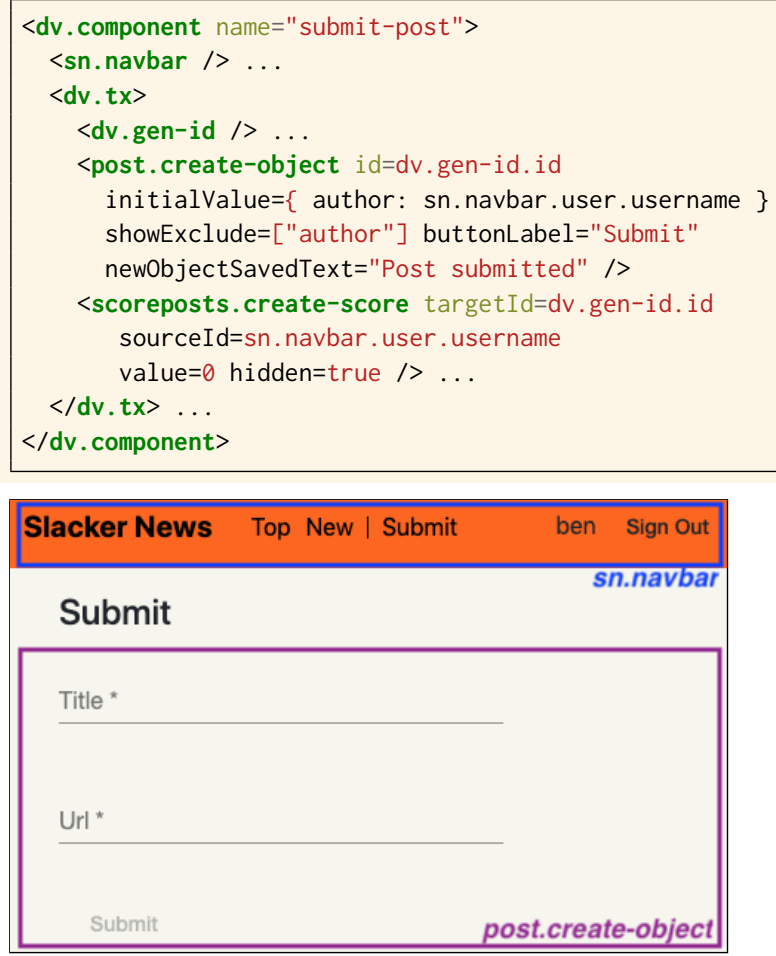

(a) Excerpt of $S N$ 's submit-post component
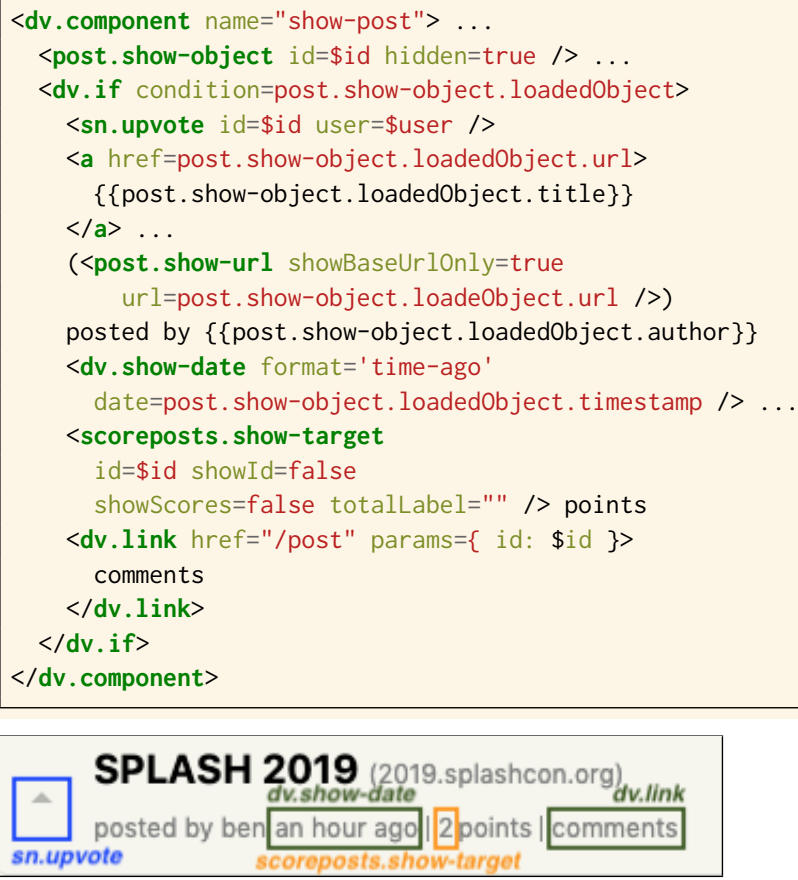

(b) Excerpt of $S N$ s show-post component

Figure 3. Linking components the object data itself is still loaded, emitted as an output, and used in several parts of the view-the title and the url are used and shown through lines 5-9, while the author is displayed on line 10 .

App components can have their own input properties. Any name used in an expression that is prefixed with $\$$ is considered to be an input property of the app component. For example, show-post has an input named id that it uses in lines 2, 4, 14, and 16 (Fig. 3b). Based on this input, show-object will show the post whose ID matches the given one; upvote will use the ID as the target of the score if one is created; show-target will show the score with the given ID; and clicking on the "comments" link will take the user to show-post-details with its input id set to the given ID.

To display how long ago the post was created, in show-post we bind the date input property of show-date to the object timestamp (line 12) and set its format input property to "time-ago" (line 11). All objects from Property have a timestamp field that stores the object's creation date.

ID Sharing. To bind entities in different concepts we use a common identifier. In submit-post (Fig. 3a), for example, the same ID, generated by gen-id (line 4), is passed to create-object (line 5) and create-score (line 9). As a result, create-score will create a score with the same target ID as the object created by create-object. Similarly, in show-post (Fig. 3b), we feed the id input to show-object (line 2) and show-target (line 14). Each of these components loads and displays its own view of the post entity; the effect when put together is to display a $S N$ post object.

\subsubsection{Synchronizing Components}

Action Types. Concept components have two actions: an evaluation action (eval) and an execution action (exec). The concept author determines what triggers the evaluation or execution of the component. Typically, the loading of the component itself triggers the evaluation of a component, and some user interaction (e.g., a button click) triggers its execution. What happens on eval or exec is also up to the author of the concept-the only restriction is that an eval action cannot produce a side effect. Note that app components don't have actions. This is because app components have no back-end functionality of their own-all data and behavior is pushed to concepts.

Eval/exec actions support the conventional user interaction pattern of web apps: data is loaded and displayed, and then the user executes commands to mutate the data. It would be possible for concept components to offer arbitrary action types to support more complex forms of behavior. But this would require more work from the user, who would now have to specify what action types are to be coordinated.

Synchronizing Actions. There are two kinds of app components: a regular component and a transaction ( $\mathrm{tx}$ ) component. A regular component allows any of its children components 


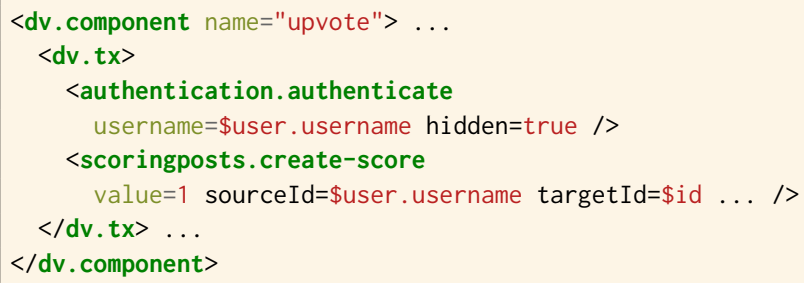

Figure 4. Excerpt of $S N$ s upvote component that shows how we prevent unauthenticated users from upvoting posts

to eval/exec without synchronization. A tx component, on the other hand, synchronizes the actions of the concept components it wraps, so that an exec in one happens with the exec of the other(s)-and similarly for eval actions. Synchronized actions either complete in their entirety if all succeed, or have no effect whatsoever other than optionally displaying an error if one or more aborts. Instead of putting each component in separate HTML files, you can wrap elements in another component with the $d v$. $t x$ tag to create an anonymous tx component with content equal to the content of the tag.

In $S N$ 's submit-post, the tx is triggered by create-object (Fig. 3a, line 5) when the user clicks on the button. This is because the button in the create-object component of Property causes the component to execute on click, and since create-object is wrapped in a dv.tx, it will trigger the execution of all its sibling concept components. As a result, a new post and a new score will be created, bound by the shared ID.

\subsection{Specifying Security Policies}

In Déjà Vu, a security policy is specified implicitly through: (1) which concepts are included and how they are configured;

(2) which components from the included concepts are used; and (3) how concept components are bound to other concept components in tx components.

For example, to implement the policy "posts must have a title" we say that the title field is required in the configuration of Property (Fig. 2, line 16). The server of Property will enforce this constraint, and return an error if no title is given when a new object is created. Other constraints, such as "posts cannot be deleted", are enforced by the omission of certain components: in this case, the delete-object component that lets you delete Property objects is not included in the app. Finally, Fig. 4 shows how we use tx components to implement the policy "only authenticated users can upvote posts". In $S N$ s upvote component, we wrap the creation of a new score in a tx component with the authenticate component of Authentication. The authenticate component checks that the logged in user matches the given username. If it doesn't, it returns an error. The error causes the transaction to abort and, as a result, it causes the upvote of a post to abort. Since there's no other component in the app that would let a user upvote a post, only authenticated users can upvote a post.

Note that policies are expressed in HTML and JSON, but, as we'll see later, are actually enforced server-side by our runtime system and concept servers.

\section{Prototype Implementation}

Déjà Vu is built using TypeScript ${ }^{9}$, Angular $^{10}$ and Node.js ${ }^{11}$. The implementation consists of:

- a front-end library to synchronize components (\$3.1);

- a server gateway to coordinate transactions and run security checks (§3.2);

- a compiler that transpiles a Déjà Vu app into an Angular app (§3.3); and

- a catalog of concepts (§3.4).

We also have a few Angular components that implement built-in Déjà Vu components such as dv.gen-id for generating IDs, dv . link for creating links, and dv. button for creating buttons that trigger the execution of a transaction.

The front-end library communicates with the gateway, which then communicates with the concept servers. The communication between a concept server and its data store requires no mediation.

\subsection{Front-End Library}

Event Dispatching. The front-end library allows concept components to register with the runtime system to get notified when they should eval/exec, and to request the system to trigger eval/execs of other components. The library is an event mediator [29]: a concept component doesn't subscribe to eval/exec events announced by other components directly, but it does so indirectly through the library. The library determines how to dispatch an eval/exec event depending on whether the app component is a tx component or not. Thus, it is as if each app component has a local mediator to coordinate its own synchronization.

Client-Server Communication. The client-side of a concept doesn't communicate with its server directly. All communication happens through the front-end library, which then communicates over HTTP to the gateway. When a component runs (evals/execs), the component tells the runtime system which inputs were provided and can give extra information for its concept server.

If the concept component being run is not part of a tx, the front-end library issues an HTTP request to the gateway as soon as the run request is received. If it is part of a tx, the front-end library triggers the eval/exec of the other concept components in the app component, batches all run requests

\footnotetext{
${ }^{9}$ https://www.typescriptlang.org/

${ }^{10}$ https://angular.io

${ }^{11}$ https://nodejs.org
} 
from all components that are part of the tx, and sends only one aggregate request to the gateway.

After the gateway processes the request, concept servers receive an HTTP request with the name of the component to run, whether it's an eval or exec, its inputs, and the extra information provided by the component.

\subsection{Gateway Server}

The communication between the gateway and concept servers happens through designated HTTP routes that all concepts are required to implement.

The gateway receives from the front-end library the information on what component executed (given as a path from the root), with what inputs, and the extra information provided by the component. At this point, the gateway runs security checks to ensure that the request is valid (more about this in \$3.2.1). If the request is invalid, it returns an error.

If the request is valid and it is a non-tx request, the gateway forwards the request to the corresponding concept server and forwards the response obtained from the concept server back to the front-end library.

If the request is a tx request, the gateway acts as a transaction coordinator and runs a two-phase commit (2PC) protocol with all the concept servers that are part of the transaction. If all concept servers vote ok, the gateway commits the tx and forwards all the responses from the concept servers in one HTTP response to the front-end library. The front-end library demultiplexes the gateway response and forwards the individual responses to the concept components. If at least one concept server votes abort, the gateway sends abort messages to all concept servers and forwards the responses from the concepts that voted abort back to the front-end library. The error responses are used client-side by concept components to show an error to the user. Note that the responses from the concept servers that voted ok are not sent back if the transaction aborts. This is to prevent a malicious client from receiving information it is not allowed to see.

\subsubsection{Security}

Our runtime system has no built-in notion of authentication or authorization. This functionality is implemented in concepts, which makes it easier for experts to author a variety of concepts that implement different authentication and authorization mechanisms, without requiring changes to the runtime system.

The server-side concept implementations are part of the trusted base, and are assumed not to have been compromised. But the client-side code, of course, cannot be trusted. We therefore need to ensure that a client cannot violate the structure of transactions, or run the server-side action of a component that is not included in the app.

There are three properties of our implementation that allow us to enforce a policy: (1) when run, components report the input values that they were run with; (2) the gateway knows what components are expected to run and what their input values could be (how it knows is explained below); and (3) concepts don't communicate with their servers directly, but rather all communication is via the gateway.

On startup, our system provides the app source code to the gateway. From the source code, the gateway builds a component tree that records the hierarchical relationships between all the components in the app and the input property bindings. When the gateway receives a request to eval/exec a certain component, it verifies that the component path argument given by the front-end library is a path of the component tree. If it isn't, it returns an error.

If the component path is a valid path, it checks that the input values given to the action are valid. If, in the app's source code, the user binds a component input to a literal, and the value doesn't match the input value given to the the action, it returns an error. For example, in $S N^{\prime}$ s upvote component (Fig. 4) the user sets the score value to 1 (value=1, line 6). As a result, when the gateway gets a request to execute the create-score action in upvote, it checks if value is 1 . Thus, a malicious user can't, e.g., add 10 points to a post in one upvote (because $10 \neq 1$ ).

If the request is a transaction request, the gateway additionally checks that the input values of all components that are part of the transaction are consistent: for every component of the transaction, the gateway evaluates the expression each input is bound to in the source code using the output values recorded at the time the tx was initiated, and checks that the result matches the given input values. For example, when the gateway gets a request to execute $S N$ 's submit-post transaction (Fig. 3a), it checks that the id input value of create-object matches the targetId value for create-score. These inputs have to match because: (1) the id input of create-object is bound to dv.gen-id.id (line 5); (2) the targetId input of create-score is bound to $d v$.gen-id.id (line 9); and (3) they are wrapped in a dv. $t x$ (lines 3-12).

\subsection{Compiler}

Our compiler outputs an Angular application from the app's config and component files. For each app component, it creates an Angular component. Our component language is a very thin layer atop Angular's template syntax.

When an app developer runs a Déjà Vu app, we run the compiler, save its output in a hidden directory, and start the gateway and the concept servers. The gateway, in addition to processing eval/exec requests, serves the Angular app generated by the compiler.

\subsection{Concept Catalog}

Table 1 shows the current state of our catalog. To give a sense of the amount of functionality implemented in each concept, we include the number of components (\# C) and the number 


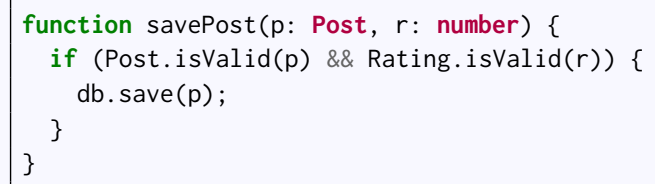

(a) Server Monolith

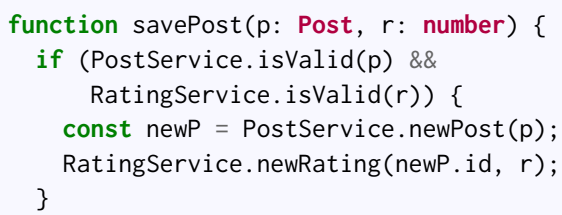

(b) Server using Microservices

Figure 6. Two server-side code variants for submit-post (monolith and microservices)

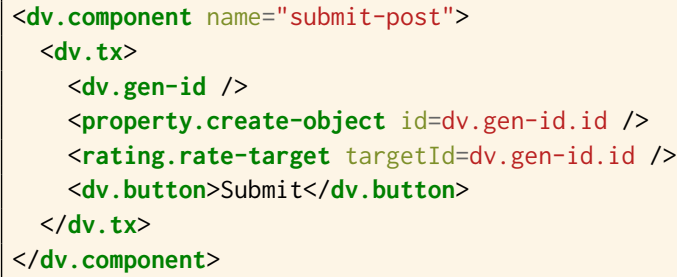

Figure 7. submit-post component in Déjà Vu

inputs (Fig. 6a or Fig. 6b, line 1). In the monolithic backend (Fig. 6a), we assume there are libraries for validating posts, ratings, and accessing the database. In the microservices back-end (Fig. 6b), we assume there are post and rating services.

Note that, even if we assume that all the post and rating functionality is readily available, there's still a lot of code that we need to write to put everything together. With a standard approach, we have to:

- Subscribe to events and write event handlers. In Angular, we subscribe to the ngSubmit event generated by the form so as to invoke the onSubmit method of the component whenever the form gets submitted (Fig. 5a, template file, line 1). In React, we subscribe to the onchange event generated by the post and rating components (Fig. 5b, lines 18-19) and to the onSubmit event of the form (Fig. 5b, line 17).

- Aggregate data for request. In Angular, the event handler for submit events, submitPost(), has to aggregate data of post-input and rating-input to build the server request. In React, the event handler for
onChange events updates the component state, which is then read by the event handler for submit events to build the server request. In both cases, the request is sent by the post service when we invoke its savePost method.

- Handle client-server communication. While we are assuming that there exists a client-side post service to issue requests, certain modifications would normally require the modification of the server API and of the client service. For example, adding a location to the post might require adding a new parameter to savePost and a new parameter to the server API so that it expects a location value.

- Combine server-side functionality. Even if the serverside functionality of posting and rating exists, we still have to, in the case of the monolith, make the appropriate function calls to validate the request and save the post to the database. Also, in the server using microservices, we still have to make the appropriate calls to the post and rating services. Moreover, integrating microservices could, in some cases, require more complex code, since one might have to implement transactions.

On the other hand, when using Déjà Vu, none of this is necessary. Fig. 7 shows the implementation of submit-post using Déjà $\mathrm{Vu}$, where we assume that we have the Property and Rating concepts. In Déjà Vu, wrapping a component in a dv.tx automatically subscribes to eval/exec events, runs the component handlers, aggregates the data client-side and sends the request, unpacks the request server-side, and coordinates the calls to the back-end services of each concept so they happen in a transaction if necessary. All of this functionality is hidden from the app developer, who doesn't need to write JavaScript code to handle events or combine serverside microservices (for example). Of course one could build a set of libraries that would alleviate the amount of integration code required when using a standard approach, but that would amount to almost replicating Déjà Vu's runtime system.

\subsection{Case Study}

We (the authors of this paper) built a small suite of applications that replicate the functionality of student projects in an undergraduate course. The student projects are from the Fall ' 16 offering of the class.

With the students' permission, we obtained access to their code repositories so we could run their applications and explore their behaviors. For each project, we developed any missing concepts in order to replicate the behavior of the original student app. We replicated only the core functionality, omitting behavioral details that are not essential to the app's working. We didn't use any of the code written by students other than some HTML to provide page content such as titles and CSS to style the appearance. 
Table 2. Student projects we replicated in Déjà Vu. The symbols next to the code count indicate the front-end library used: ${ }^{\dagger}$ React v15, ${ }^{*}$ Handlebars v4, ${ }^{* *}$ Jade v1

\begin{tabular}{lll}
\hline App & Purpose & LoC \\
\hline Accord & Support musical bands in the selection of setlists & $8,671^{\dagger}$ \\
ChoreStar & Make it easy for parents to assign chores to children & $3,183^{*}$ \\
EasyPick & Recommend classes to college students & $3,1^{*}$ \\
GroceryShip & Facilitate peer grocery delivery between students & $4,996^{*}$ \\
Lingua & Develop language skills by chatting with native speakers & $4,639^{\dagger}$ \\
Listify & Crowdsource opinion-based rankings of anything & $5,876^{\dagger}$ \\
LiveScorecard & Provide a live leaderboard for climbing competitions & $8,742^{\dagger}$ \\
MapCampus & Allow students to plan events on campus & $3,807^{\dagger}$ \\
Phoenix & Help people discuss mental health and make friends & $7,062^{*}$ \\
Potluck & Help people plan parties where guests bring supplies & $4,344^{\dagger}$ \\
Rendezvous & Plan public events on campus & $4,498^{* *}$ \\
SweetSpots & Mark spots on a map and review spots added by others & $3,898^{\dagger}$ \\
\hline
\end{tabular}

The 12 apps we replicated were selected independently by the teaching assistants of the class as the best projects out of about 30 projects. The project selection happened before we contacted students about using their projects to evaluate Déjà $\mathrm{Vu}$, and the teaching assistants of the course have no relation to our research project.

The names of the student projects we replicated, together with their purposes, are shown in Table 2. We also include the number of lines of HTML, CSS, client- and server-side JavaScript code for the student implementations. ${ }^{13}$ The student projects were mostly 4-person projects, done for 5 weeks, with each student taking 10-20 hours per week. Thus, each project represents 200-400 person-hours of work.

Through the case study, we sought to answer two research questions:

- Is it possible to build a variety of non-trivial applications using Déjà Vu? (§4.2.1)

- Can this be done without building non-generic concepts that are specific only to a given app? (\$4.2.2)

\subsubsection{Experience}

Selecting Concepts. Selecting a concept to provide required functionality is relatively easy, since concepts have mostly non-overlapping functionality. A possible source of overlap we have experienced is due to the flexibility of Property. For example, in Chorestar, parents have to be associated with the children accounts they create. This is so that, for example, parents can assign chores only to their children and not to other children in the system. There are two ways to do this. One way is to use Group, and compose it in such a way that when a parent account is created, a group with the same ID is created as well. Then, when a new child account is created, the child is added as a member of the group with the same ID as the ID of the logged in parent. The other option is to

\footnotetext{
${ }^{13}$ The count includes comments and blank lines. Unit tests are not counted.
}

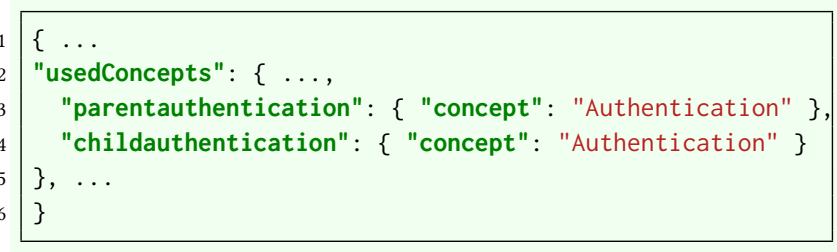

(a) Excerpt of the configuration file for ChoreStar

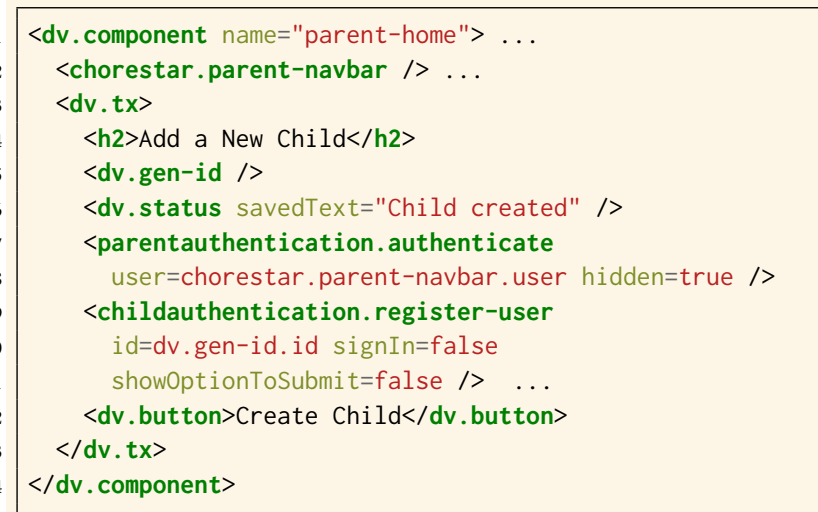

(b) Excerpt of parent-home

Figure 8. Excerpts of ChoreStar

use Property, and configure it so that child profiles have a field parentId. Then, when a new child account is created, you create a child object with the parentId field set to the ID of the logged in parent. In a case like this, one could use either Property or Group to implement the desired behavior.

With the exception of cases like this, selecting concepts is straightforward. In the future, however, as the catalog grows, selecting a concept might be harder. Especially if the catalog becomes more like a marketplace, in which there might be multiple variants of each concept. In this case, selecting a concept would be more akin to choosing a theme in a CMS such as WordPress.

Implementing Complex Behaviors. Non-trivial behavior that might, at first, seem like it would require a very specific concept, can often be implemented by combining existing concepts. For example, in ChoreStar, there are two kinds of users, parents and children, and only parents can create children accounts. This relatively uncommon feature was easy to implement in Déjà Vu (Fig. 8): we included Authentication twice (Fig. 8a), and synchronized the register-user component for children with the authenticate component of parents (Fig. 8b).

Incremental Development. With Déjà $\mathrm{Vu}$, it is possible to develop an app incrementally. We found that this made it easier to evolve and debug applications as we built them. For example, in Rendezvous, campus events have a location and 
a guest-list that only hosts can edit, but everyone else can view. In Déjà $\mathrm{Vu}$, you can start with the event and location functionality by including Event and Geolocation, check that it works as you expect, then add guests with Group, and so on, one concept at a time.

Protecting Actions. We noticed that it is easy to forget to protect actions with authenticate, especially because authenticate produces no visible change in the behavior of the app. In most apps, users have to sign in, after which they are redirected to some other page where they can perform operations only authenticated users are supposed to perform. For example, in Chorestar, after a parent signs in, the parent is redirected to the parent home, where it can create chores. During manual testing, it is easy to assume that the actions are protected, since you can only reach that page using normal traversal of the site if you are already signed in. But if the authenticate component is not wrapped in a dv.tx with the component it is supposed to protect, a malicious user can craft an HTTP request to perform the action without authenticating. A potential solution to this problem is to analyze the app's source code and, in the style of [21], warn the user if we find e.g., a create-score action protected, but a delete-score not.

App Freeze. It is possible to write Déjà Vu code that causes the app to freeze. This is more likely to happen in Déjà $\mathrm{Vu}$ than in app development in general, because actions can block when they are triggered externally and don't have the inputs they need to run. This could happen if, for example, the user forgets an I/O binding, or if the output of a component that is produced after a tx is bound to a required input of another component in the same tx. This is less of a problem than it might at first appear to be: unlike the authentication problem discussed above, it is easy to realize that something is wrong with the app because it freezes on every test run. To help the app developer, one could perhaps incorporate something like session types [16] to detect statically if a required input was not given or if there is a deadlock.

\subsubsection{Modularity Analysis}

In this section, we analyze how the apps we built to replicate student projects use the catalog. The apps in the suite, together with the number of times they use a concept from the catalog, are shown in Table 3.

Discussion. The median number of concept types used per app is $Q_{2}=6\left(Q_{1}=5.75, Q_{3}=8\right)$. The median number of concept instances used per app is $Q_{2}=9\left(Q_{1}=7.75, Q_{3}=10\right)$. Most apps use roughly the same number of concept instances $(\sigma=2.3)$. This is probably because all the student projects we replicated took a similar amount of person-hours of work to develop, and are therefore roughly equivalent in terms of complexity.

The median number of times a concept is used across projects is $Q_{2}=3\left(Q_{1}=1, Q_{3}=5\right)$. The median number of times
Table 3. Concept usage in sample apps

\begin{tabular}{|c|c|c|c|c|c|c|c|c|c|c|c|c|c|c|}
\hline Concept/App & $\begin{array}{l}\overrightarrow{0} \\
\stackrel{0}{0} \\
\dot{\psi}\end{array}$ & 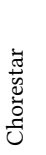 & 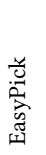 & 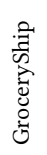 & 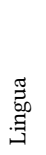 & 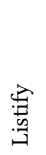 & 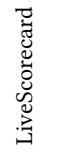 & 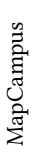 & 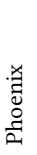 & 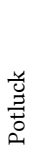 & 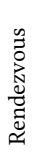 & 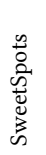 & 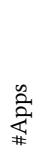 & 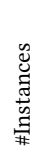 \\
\hline Authentication & 1 & 2 & 1 & 1 & 1 & 1 & 1 & 1 & 1 & 1 & 1 & 1 & 12 & 13 \\
\hline Authorization & 1 & 1 & 1 & 1 & 1 & 1 & 1 & 2 & 1 & 1 & 1 & 1 & 12 & 13 \\
\hline Chat & 0 & 0 & 0 & 0 & 1 & 0 & 0 & 0 & 0 & 0 & 0 & 0 & 1 & 1 \\
\hline Comment & 1 & 0 & 1 & 0 & 0 & 0 & 0 & 0 & 1 & 0 & 1 & 1 & 5 & 5 \\
\hline Event & 0 & 0 & 0 & 0 & 0 & 0 & 1 & 1 & 0 & 1 & 1 & 0 & 4 & 4 \\
\hline Follow & 0 & 0 & 0 & 0 & 0 & 0 & 0 & 0 & 0 & 0 & 0 & 1 & 1 & 1 \\
\hline Geolocation & 0 & 0 & 0 & 0 & 0 & 0 & 0 & 1 & 1 & 0 & 1 & 1 & 4 & 4 \\
\hline Group & 1 & 0 & 0 & 0 & 2 & 1 & 3 & 1 & 0 & 1 & 1 & 0 & & 10 \\
\hline Label & 0 & 0 & 0 & 0 & 0 & 0 & 0 & 0 & 1 & 0 & 1 & 1 & 3 & 3 \\
\hline Match & 0 & 0 & 0 & 0 & 0 & 0 & 0 & 0 & 1 & 0 & 0 & 0 & 1 & 1 \\
\hline Passkey & 0 & 0 & 0 & 0 & 0 & 0 & 2 & 0 & 0 & 0 & 0 & 0 & & 2 \\
\hline Property & 5 & 3 & 3 & 3 & 2 & 2 & 4 & 3 & 2 & 2 & 2 & 1 & & 32 \\
\hline Ranking & 0 & 0 & 0 & 0 & 0 & 1 & 0 & 0 & 0 & 0 & 0 & 0 & 1 & 1 \\
\hline Rating & 1 & 0 & 4 & 1 & 1 & 0 & 0 & 0 & 0 & 0 & 0 & 1 & 5 & 8 \\
\hline Schedule & 0 & 0 & 0 & 0 & 0 & 0 & 0 & 0 & 1 & 0 & 0 & 0 & 1 & 1 \\
\hline Scoring & 0 & 0 & 0 & 0 & 0 & 1 & 2 & 0 & 0 & 0 & 0 & 2 & 3 & 5 \\
\hline Task & 0 & 1 & 0 & 0 & 0 & 0 & 1 & 0 & 0 & 0 & 0 & 0 & 2 & 2 \\
\hline Transfer & 0 & 1 & 0 & 0 & 0 & 0 & 0 & 0 & 0 & 1 & 0 & 0 & 2 & 2 \\
\hline \# Concept Types & 6 & 5 & 5 & 4 & 6 & 6 & 8 & 6 & 8 & 6 & 8 & 9 & & \\
\hline \# Concept Instances & 10 & 8 & 10 & 6 & 8 & 7 & 15 & 9 & 9 & 7 & 9 & 10 & & \\
\hline
\end{tabular}

a concept is instantiated is $Q_{2}=3.5\left(Q_{1}=1.25, Q_{3}=7.25\right)$. All of the apps require identification of users and preventing them from accessing content they don't own. Also, it is very common for apps to need to store domain-specific fields. For example, a "description" for parties in Potluck. Thus, Authentication, Authorization, and Property are the most used concepts. The Property concept is the concept with the largest number of instances. This is because apps need an instance of Property for each kind of entity, and a given app could have multiple entities. For example, in Accord, there are bands, song suggestions, setlists, media links, and user profiles.

Chat, Follow, Match, Ranking, and Schedule are only used once. We do not think it is because these concepts are too application-specific. For Chat, we think it might be because it is challenging to implement, so only one of the winning student teams risked doing so. With enough time, other projects might have ended up incorporating such functionality. For example, Rendezvous might have created a group chat for each campus event so that guests could talk.

Follow implements functionality that is very common in social media applications: subscribing to a source of updates. For example, Twitter lets you follow other accounts, and tweets from accounts you follow appear in your feed. Ranking lets users rank items and show the aggregate consensus ranking of items. While this looks like a rather specific concept, note that many apps for managing human resources usually include functionality like this, so that managers and 
stakeholders can stack rank employees to determine promotions.

Match and Schedule are only used in Phoenix. Phoenix's functionality revolves around matching users after they both expressed interest in each other and giving a way for users that match to find a time to meet in person. While no other sample app uses Match or Schedule, many apps, such as dating sites, have matching functionality. And many productivity apps provide functionality for scheduling meetings.

Deviations. We noticed that it becomes evident when some project deviates from what you'd expect the normal behavior of certain functionality to be. For example, in Phoenix, when two users match because they have expressed an interest in meeting each other, the app lets the user write a message to their match. You would expect the app to let users send messages back and forth with their match from within the app, but it doesn't. The message is written within the app, but it is sent via email, and no record of the message is left in the storage of the app itself. This behavior is not well supported by a concept. One could, of course, replicate this functionality by simply implementing a concept for sending emails, but a concept that implements a messaging system would make more sense. In Phoenix, one could then combine this messaging concept concept with a notification concept so as to notify users via email when there's a new message in their inbox.

Perhaps the students wanted to build a messaging system within the app but didn't have enough time, so they ended up with functionality that is almost a message inbox with email notifications, but not quite. Or perhaps this is what they intended to do in the first place. Either way, it raises an interesting research question-which we have yet to exploreabout whether deviations from the norm (where "norm" is functionality that can be built by combining concepts Déjà Vu-style) represent design flaws in the application or the invention of a novel concept.

Criteria for Creating Concepts. To avoid overlapping functionality between concepts, we only add a new concept to the catalog if there is no other concept with a similar purpose, and we only add functionality to a concept if such functionality cannot be obtained by combining the concept with other concepts. But having simpler and more orthogonal concepts can mean more work combining them. For example, Authentication does not include assigning first and last names to users, since this functionality can be obtained by adding Property. It would be easier for app developers, however, to have such common features included in Authentication as a configuration option despite the redundancy. The right balance will have to be found empirically.

A different question is whether it is desirable for the catalog to contain multiple variants of a single concept, for example a variant of Authentication in which an email address it the primary identifier versus one in which a social security number is used. Put another way, is the catalog more like a marketplace (as found with WordPress themes, for example) or is it more like a polished standard library (as with java.util)? This remains to be seen.

\section{Related Work}

\subsection{Programming Paradigms}

Object-Oriented Programming (OOP). Concepts and their instances are roughly analogous to classes and objects. But the components of a concept, unlike the methods of an object, have full-stack implementations that include visual representations and interaction widgets.

The composition of different concepts could be seen as including the behavior associated with one concept in the other (and vice versa), and thus has some similarities to mixins $[5,10]$ and traits $[8,26]$. But in Déjà $V u$, the extra behavior is not necessarily orthogonal to the existing behavior. Synchronizing components intertwines these behaviors: running a concept action might also trigger some of the included behavior.

Subject-Oriented Programming (SOP). In SOP [15], a subject is a collection of states and behaviors reflecting a particular view. Each subject can separately define and operate upon shared objects, without any subject needing to know the details associated with those objects by other subjects.

A subject is like a concept, but SOP is concerned with the decomposition of the same object into different perceptions (subjects). In Déjà Vu, concepts are, a priori, not talking about the same objects at all. It is only after they are composed together that one can see concepts as providing different views over the same entities connected by bindings.

Moreover, in SOP, subjects are composed via a composition rule, which can specify arbitrary requirements for the composition, and require the implementation of a subject compositor (code that combines subjects in an environment according to the rules). Adding new subjects to a composition requires adding new rules and modifying the subject compositor. In Déjà $\mathrm{Vu}$, the user determines the composition rule and the subject compositor by deciding whether the containing component is a tx or regular component, and by the property bindings.

Aspect-Oriented Programming (AOP). In AOP [18], the goal is to increase modularity by allowing the separation of cross-cutting concerns such as logging. The AOP approach is to separate a program into "core concerns" that implement the basic functionality of the software, and "crosscutting concerns" (aspects) that encapsulate functionality that is shared by multiple core concerns. Aspects alter the behavior of core concerns by applying additional behavior (advice) at various points in the program (join points).

Viewed through an AOP lens, the concepts of a Déjà Vu app are usually all core concerns. If there are join points, 
they would be implicit in the synchronization of the tx components.

Feature-Oriented Programming (FOP). In FOP, a program is a base program plus a stack of features [3]. While a concept might be viewed as a kind of feature, not every feature is a concept. A feature may represent an entire collection of concepts. For example, the "news feed" feature on Facebook includes concepts such as "feed", "comment", and "likes". Or a feature may represent a small increment of functionality that would be part of a concept. For example, a "password validation feature", which would be part of the "authentication" concept.

Also, features do not generally exist independently of the base, and are included in a predefined way. For example, in AHEAD [4], features are nested tuples of program deltas. When applied to a program, the source code is transformed by applying the delta.

Event-Driven Programming. In event-driven programming [13], software components can publish or subscribe to events, and the flow of the program is determined by these events. New software components can add behavior to a system by subscribing to a particular event, without requiring the modification of the software component that publishes it. Our implementation of Déjà $\mathrm{Vu}$ is event driven: concept components announce eval/exec events and are notified when it is time for them to eval/exec. But this is hidden from the app developer; app components can't announce events or have concept components listen to arbitrary events.

Postmodern Programming. Our approach could be seen as an instance of postmodern programming [23, 24] in that the programming effort involves primarily gluing existing parts together rather than creating new ones. Contrary to other postmodern approaches, however, it should be noted that our composition mechanism and language are homogeneous. The heterogeneity of component implementations is encapsulated and not visible to the end-user programmer.

Behavioral Programming (BP) In BP [14], an app consists of independent modules that run in parallel and communicate via events. Modules in BP (behaviors) are more granular than concepts, because there's usually one per software requirement, while a single concept would support multiple requirements. In $\mathrm{BP}$ and Déjà $\mathrm{Vu}$, modules can trigger, subscribe to, and block events. In Déjà $\mathrm{Vu}$, however, blocking an event is not something the user can specify as a means of controlling the flow of the program, but happens automatically when a component action fails.

\subsection{Architectural Patterns}

Microservices. Microservices is a popular architectural style that structures an application as a collection of loosely-coupled software services that are independently deployable [22]. Microservices is an approach to service-oriented architecture
(SOA) [9] that emphasizes building services around business capabilities and using lightweight communication mechanisms like HTTP.

A business capability usually involves more than one concept. Therefore, a microservice tends to aggregate more functionality than a concept. For example, an e-commerce site using microservices might have a customer feedback service that aggregates together reviews and ratings, while in Déjà $\mathrm{Vu}$, reviews and ratings would be separate concepts.

Another difference is that, in practice, microservices provide back-end functionality only. Even in those cases in which microservices are full stack, ${ }^{14,15}$ developers have to write complex code to coordinate between different services. In Déjà $\mathrm{Vu}$, the developer has only to specify what actions need to occur in a transaction, and Déjà Vu will take care of coordinating between the different concept back-ends.

Déjà $\mathrm{Vu}$ can thus be viewed as an attempt to realize a microservices architecture with full-stack microservices that are more granular, easier to combine, and generic enough to be reused in multiple applications or in a single application multiple times.

Entity-Component-System (ECS). ECS [1] is an architectural pattern used in the development of computer games and other real-time interactive systems. In ECS, an entity (ID) is partitioned into multiple components (the raw data of one aspect of the entity). The code that implements certain functionality is located in a system, which can operate on multiple components. A concept component is like an ECS's system in the sense that it implements certain functionality that operates on the raw data of one aspect of the entity. But, unlike a system, a concept component can only interact with one aspect of the entity (because it can't communicate with other concepts). App components are perhaps more like ECS's systems (because they can operate on more than one aspect of the entity), but an app component, unlike a system, can't operate on the raw data of one aspect directly-it can only do so through concept components.

\subsection{CMSs and Low-Code Platforms}

As discussed in $\$ 1.2$, the plug-ins of CMSs and low-code platforms lack a generic composition mechanism. While plug-ins are full-stack like concepts, getting different plugins to work together can require a lot of effort.

\subsection{Web Frameworks}

There are many software frameworks and libraries to support web development. Some focus on the client-side development (e.g., Angular, React and Vue ${ }^{16}$ ) and others on the server

\footnotetext{
${ }^{14} \mathrm{https}: / /$ micro-frontends.org/

${ }^{15} \mathrm{http}: / / \mathrm{scs}$-architecture.org/

${ }^{16}$ https://vuejs.org/
} 
side (e.g., Rails ${ }^{17}$ and Django ${ }^{18}$ ). Full-stack frameworks (e.g., Meteor ${ }^{19}$ ) and tierless web programming languages (e.g., $[6,7,27])$ provide support for both.

The essential difference between web frameworks and Déjà $\mathrm{Vu}$, is that web frameworks are designed to be general purpose and require you to write all the logic of end-user behavior yourself. The benefit is that any app can be built using web frameworks (not just what can be built with the catalog). The drawback is that each concept has to be implemented anew. Even if full-stack components implementing a particular concept exist (e.g., those provided by Disqus for comments), or if some elements of a concept implementation can be obtained from a library, you still have to write complex client- and server-side code to fill in the missing parts, or to integrate the functionality with the rest of the app.

Our template language is, by design, similar to popular template languages like the one used in Angular or React's JSX. For example, components are included by name as if they were standard HTML elements; and the user can bind an expression to an input, which will recompute and update the target input property when data changes. The difference lies in the fact that Déjà $\mathrm{Vu}$ components have an eval and exec action, and that there are different types of components ( $t x$ and non-tx) that the user can create to determine behaviorwithout having to write any JavaScript.

\subsection{Design Patterns}

Concepts provide a recipe for implementing a solution that satisfies a particular purpose. In this sense, they are related to Alexander's design patterns [2], analysis patterns [11], or the more implementation-centric patterns of OOP [12]. Unlike these, concepts not only describe a solution but they make it tangible: they embody the design pattern, and can be readily executed and combined.

The programmer's apprentice project [25] includes a catalog of commonly recurring structures in code, requirements, or other phases of software development. These structures, like our concepts, capture and implement a common pattern. But our concepts capture higher-level aggregations of behavior.

\section{Conclusion and Future Work}

Déjà $\mathrm{Vu}$ is a new platform for assembling web apps by configuring and composing concepts drawn from a catalog. Our work so far has demonstrated the viability of our approach to build web applications. The concept composition mechanism is simple and requires writing no procedural code.

Moreover, the configuration and composition of concepts is amenable to graphical representations and suitable for a

\footnotetext{
${ }^{17}$ https://rubyonrails.org/

${ }^{18}$ https://www.djangoproject.com/

${ }^{19}$ https://www.meteor.com/
}

graphical programming environment since concept components are GUI elements. Together with the development of the platform, we have been developing such an environment. Coupled with a rich catalog, a graphical environment for Déjà Vu could allow app developers to build applications with rich behavior, without writing any binding or configuration code at all.

\section{Acknowledgments}

Thank you to our anonymous reviewers and to our shepherd, Thomas LaToza, whose insightful critique and guidance greatly improved this paper. Thanks also to the undergraduate students that contributed to our project through MIT's UROP program: Yunyi Zhu, Shinjini Saha, John Parsons, Stacy Ho, Teddy Katz, and Eric Manzi. This research was funded in part by the International Design Center, a collaboration between MIT and SUTD (the Singapore University of Technology and Design).

\section{References}

[1] T. Alatalo. 2011. An Entity-Component Model for Extensible Virtual Worlds. IEEE Internet Computing 15, 5 (Sep. 2011), 30-37. https: //doi.org/10.1109/MIC.2011.82

[2] C. Alexander, S. Ishikawa, and M. Silverstein. 1977. A Pattern Language: Towns, Buildings, Construction. Oxford University Press USA.

[3] Sven Apel, Don Batory, Christian Kstner, and Gunter Saake. 2013. Feature-Oriented Software Product Lines: Concepts and Implementation. Springer Publishing Company, Incorporated.

[4] Don Batory, Jacob Neal Sarvela, and Axel Rauschmayer. 2003. Scaling Step-wise Refinement. In Proceedings of the 25th International Conference on Software Engineering (ICSE '03). IEEE Computer Society, Washington, DC, USA, 187-197. http://dl.acm.org/citation.cfm?id= 776816.776839

[5] Gilad Bracha and William Cook. 1990. Mixin-based Inheritance. In Proceedings of the European Conference on Object-oriented Programming on Object-oriented Programming Systems, Languages, and Applications (OOPSLA/ECOOP '90). ACM, New York, NY, USA, 303-311. https: //doi.org/10.1145/97945.97982

[6] Adam Chlipala. 2015. Ur/Web: A Simple Model for Programming the Web. In Proceedings of the 42Nd Annual ACM SIGPLAN-SIGACT Symposium on Principles of Programming Languages (POPL '15). ACM, New York, NY, USA, 153-165. https://doi.org/10.1145/2676726.2677004

[7] Ezra Cooper, Sam Lindley, Philip Wadler, and Jeremy Yallop. 2007. Links: Web Programming Without Tiers. In Proceedings of the 5th International Conference on Formal Methods for Components and Objects (FMCO'06). Springer-Verlag, Berlin, Heidelberg, 266-296. http://dl. acm.org/citation.cfm?id=1777707.1777724

[8] Stéphane Ducasse, Oscar Nierstrasz, Nathanael Schärli, Roel Wuyts, and Andrew P Black. 2006. Traits: A mechanism for fine-grained reuse. ACM Transactions on Programming Languages and Systems (TOPLAS) 28, 2 (2006), 331-388.

[9] Thomas Erl. 1900. Service-oriented architecture: concepts, technology, and design. Pearson Education India.

[10] Matthew Flatt, Shriram Krishnamurthi, and Matthias Felleisen. 1998. Classes and Mixins. In Proceedings of the 25th ACM SIGPLAN-SIGACT Symposium on Principles of Programming Languages (POPL '98). ACM, New York, NY, USA, 171-183. https://doi.org/10.1145/268946.268961

[11] Martin Fowler. 1997. Analysis patterns: reusable object models. AddisonWesley Professional. 
[12] Erich Gamma, Richard Helm, Ralph Johnson, and John Vlissides. 1995. Design Patterns: Elements of Reusable Object-oriented Software. AddisonWesley Longman Publishing Co., Inc., Boston, MA, USA.

[13] David Garlan and David Notkin. 1991. Formalizing design spaces: Implicit invocation mechanisms. In VDM'91 Formal Software Development Methods. Springer, Springer Berlin Heidelberg, Berlin, Heidelberg, 31-44.

[14] David Harel, Assaf Marron, and Gera Weiss. 2012. Behavioral Programming. Commun. ACM 55, 7 (July 2012), 90-100. https://doi.org/ $10.1145 / 2209249.2209270$

[15] William Harrison and Harold Ossher. 1993. Subject-oriented Programming: A Critique of Pure Objects. In Proceedings of the Eighth Annual Conference on Object-oriented Programming Systems, Languages, and Applications (OOPSLA '93). ACM, New York, NY, USA, 411-428. https://doi.org/10.1145/165854.165932

[16] Kohei Honda, Vasco T. Vasconcelos, and Makoto Kubo. 1998. Language primitives and type discipline for structured communication-based programming. In Programming Languages and Systems, Chris Hankin (Ed.). Springer Berlin Heidelberg, Berlin, Heidelberg, 122-138.

[17] Daniel Jackson. 2015. Towards a Theory of Conceptual Design for Software. In 2015 ACM International Symposium on New Ideas, New Paradigms, and Reflections on Programming and Software (Onward!) (Onward! 2015). ACM, New York, NY, USA, 282-296. https://doi.org/ $10.1145 / 2814228.2814248$

[18] Gregor Kiczales, John Lamping, Anurag Mendhekar, Chris Maeda, Cristina Lopes, Jean-Marc Loingtier, and John Irwin. 1997. Aspectoriented programming. In ECOOP'97 - Object-Oriented Programming, Mehmet Akşit and Satoshi Matsuoka (Eds.). Springer Berlin Heidelberg, Berlin, Heidelberg, 220-242.

[19] Matt McCutchen, Shachar Itzhaky, and Daniel Jackson. 2016. Object Spreadsheets: A New Computational Model for End-user Development of Data-centric Web Applications. In Proceedings of the 2016 ACM International Symposium on New Ideas, New Paradigms, and Reflections on Programming and Software (Onward! 2016). ACM, New York, NY, USA, 112-127. https://doi.org/10.1145/2986012.2986018

[20] M Douglas McIlroy. 1968. Mass-produced software components. In Proceedings of the 1st International Conference on Software Engineering, Garmisch Pattenkirchen, Germany. 88-98.
[21] Joseph P. Near and Daniel Jackson. 2016. Finding Security Bugs in Web Applications Using a Catalog of Access Control Patterns. In Proceedings of the 38th International Conference on Software Engineering (ICSE '16). ACM, New York, NY, USA, 947-958. https://doi.org/10.1145/2884781. 2884836

[22] Sam Newman. 2015. Building Microservices (1st ed.). O'Reilly Media, Inc.

[23] James Noble and Robert Biddle. 2002. Notes on postmodern programming. In Proceedings of the Onward Track at OOPSLA, Vol. 2. 49-71.

[24] James Noble and Robert Biddle. 2004. Notes on Notes on Postmodern Programming. SIGPLAN Not. 39, 12 (Dec. 2004), 40-56. https://doi. org $/ 10.1145 / 1052883.1052890$

[25] Charles Rich and Richard C. Waters. 1988. The Programmer's Apprentice: A Research Overview. Computer 21, 11 (Nov. 1988), 10-25. https://doi.org/10.1109/2.86782

[26] Nathanael Schärli, Stéphane Ducasse, Oscar Nierstrasz, and Andrew P Black. 2003. Traits: Composable units of behaviour. In ECOOP 2003 Object-Oriented Programming, Luca Cardelli (Ed.). Springer, Springer Berlin Heidelberg, Berlin, Heidelberg, 248-274.

[27] Manuel Serrano and Vincent Prunet. 2016. A Glimpse of Hopjs. In Proceedings of the 21st ACM SIGPLAN International Conference on Functional Programming (ICFP 2016). ACM, New York, NY, USA, 180-192. https://doi.org/10.1145/2951913.2951916

[28] Guy L Steele. 1999. Growing a language. Higher-Order and Symbolic Computation 12, 3 (1999), 221-236.

[29] Kevin J. Sullivan and David Notkin. 1992. Reconciling Environment Integration and Software Evolution. ACM Trans. Softw. Eng. Methodol. 1, 3 (July 1992), 229-268. https://doi.org/10.1145/131736.131744

[30] Lea Verou, Amy X. Zhang, and David R. Karger. 2016. Mavo: Creating Interactive Data-Driven Web Applications by Authoring HTML. In Proceedings of the 29th Annual Symposium on User Interface Software and Technology (UIST '16). ACM, New York, NY, USA, 483-496. https: //doi.org/10.1145/2984511.2984551 